This item was submitted to Loughborough's Research Repository by the author.

Items in Figshare are protected by copyright, with all rights reserved, unless otherwise indicated.

\title{
Using eye gaze in intelligent interactive imaging training
}

PLEASE CITE THE PUBLISHED VERSION

http://dx.doi.org/10.1145/2002333.2002340

PUBLISHER

(c) ACM

VERSION

AM (Accepted Manuscript)

LICENCE

CC BY-NC-ND 4.0

REPOSITORY RECORD

Chen, Yan, and Alastair G. Gale. 2019. "Using Eye Gaze in Intelligent Interactive Imaging Training". figshare. https://hdl.handle.net/2134/20210. 


\section{Using Eye Gaze in Intelligent Interactive Imaging Training}

\author{
Yan Chen \\ Applied Vision Research Centre, \\ Loughborough University, UK \\ y.chen3@lboro.ac.uk
}

\author{
Alastair Gale \\ Applied Vision Research Centre, \\ Loughborough University, UK \\ a.g.gale@lboro.ac.uk
}

\begin{abstract}
Medical imaging, particularly in breast cancer screening, requires very skilled interpretation only carried out by specially trained radiologists. A key issue is how to train such skilled behaviour? Recent changes to breast imaging has seen the introduction of high resolution digital imaging which facilitates intelligent interactive training. It has also enabled potential computer aided detection of abnormalities. However, this also tends to increase false positive cancer detections. A series of experiments are reported which examine the role of eye gaze and expertise in inspecting these images. It is proposed that current training approaches could be augmented by including aspects of the eye gaze behaviour of expert screening radiologists together with computer aided detection in new practical interactive training systems.
\end{abstract}

\section{Author Keywords}

Eye gaze, intelligent interactive training, breast screening training.

\section{ACM Classification Keywords}

Information interfaces and presentation (e.g., HCI), Computer in life and medical science.

\section{INTRODUCTION}

It is well established in image inspection tasks that experienced observers exhibit measurable differences in visual search strategy as compared to domain inexperienced individuals. A body of research has investigated the underlying reasons for this and how such differences are developed. That expert performance relates to specific visual search behaviour implies that potentially the visual search behaviour of experts can be utilised in some way to improve the performance of the less experienced, either in general training or in accelerating existing training programmes.

Presented at International IUI 2010 Workshop on Eye Gaze in Intelligent Human Machine Interaction.
Much of this research has been performed in the domain of medical image inspection and builds upon theoretical foundations which posit the importance of visual search as a key part of the complex development of skilled cognitive performance [1]. Early research largely concentrated upon the chest X-ray image but recently most work has investigated mammographic image interpretation which involves the examination of screening mammograms for very early signs (often only a few millimetres in size) of breast cancer.

\section{BREAST CANCER SCREENING}

In breast screening, each breast is now generally imaged twice (both the Media-Lateral Oblique [MLO] view and the Crania-Caudal $[\mathrm{CC}]$ view) from different angles, so as to overcome perceptual ambiguities. Therefore, a screened woman is represented by four images. To identify potential cancerous signs then (1) each single image has to be examined separately in detail, as well as (2) comparing the two different images, MLO and CC, of each breast and (3) comparing one breast image with the similar image (e.g. MLO) of the contra-lateral breast.

In the UK, there has been a recent move from using X-ray film, which is then examined on an illuminated multi-viewer, to using digital imaging of the breast with examination of the resultant images on very high resolution digital workstations. In order to examine fine detail in mammograms, radiologists used to use a magnifying glass with the X-ray film mammograms, whereas now they can utilize numerous software interaction tools.

Additionally Computer Aided Detection (CAD), where algorithms help identify suspicious image areas on the digital images, has been developed and is being introduced in some UK centres as an intelligent aid to the screening radiologist. CAD approaches work by offering the observer prompts indicating regions of an image which the CAD algorithms consider are indicative of abnormality. Typically in the past such CAD systems have produced many false positive detections but recent advances have improved on this. A recent large study compared performance when two screeners examined cases as compared to a single screener with a CAD system and found similarity between the two approaches in terms of performance [2]. However the CAD approach produced a small but significant increase in recall rates (i.e. false positive decisions). 
Errors occur in every image interpretation domain and in breast screening error occurrence, particularly false negative decisions of there being no cancer present, when in fact cancer is present, are particularly serious. For instance, recently (September, 2009) in the UK one radiologist was found to have missed cancer in 14 women, with another 85 needing to be re-imaged and a further 355 women's mammograms requiring re-examination by an expert. Minimising the potential for error by improving performance through better training is therefore very important.

\section{CURRENT TRAINING}

Current training regimes comprise courses, text books and elearning where abnormality appearances are shown, highlighted, demarcated and described; with interactive training producing feedback to the participant. Typically either the abnormal area is shown within the whole breast image or the abnormality is shown magnified. Such approaches train observers by familiarising them with a range of abnormal and normal appearances, demonstrating what to look for as well as indicating potential high probability areas within the mammographic images of where to look for abnormality. Additionally, in the UK Breast Screening Programme every screener has to examine images of at least 5,000 women a year to help develop and maintain their appreciation of such appearances.

\section{EFFECT OF DOMAIN KNOWLEDGE}

Clearly experienced observers have considerable domain knowledge which aids their performance and this must affect their visual inspection behaviour of these images. To examine the effect of little domain knowledge on eye gaze a series of MLO mammographic images were presented to a group of radiography students who had knowledge of X-ray image appearances but little specialized mammographic experience [3].

Images were presented on a 20 " LCD monitor and eye movements were recorded using a Tobii X50 eye-tracker (Figure 1). They were first familiarised through a short training session with the appearance of two key mammographic features - masses and calcifications (respectively a relatively large abnormality type, and a very small abnormality). The task required them to examine each case and identify if there was any abnormality present. If they suspected an abnormality, they also had to specify the abnormal feature type and its location. Overall only some $18.7 \%$ of responses correctly identified the cases and specified location for the abnormal case; for the normal cases the percentage of correct answer was below $41.7 \%$. These data indicate that by simply presenting key domain knowledge alone it was difficult for them to perform well. Eye movement records indicated that they spent a long time examining only certain breast areas (Figures 2 and 3) which underlies their poor performance.

\section{EYE GAZE AND BREAST SCREENING TRAINING}

Could eye gaze then have a role to play in such training? A key expert radiologist [4] has advocated the importance of visually comparing the MLO images together and has argued the importance of using a mechanical device to limit the overall visual field so as to enable improved concentration on comparing similar image areas from each breast. Consequently, actual eye movement records of individuals examining screening images can therefore be studied for the number of 'cross-over' movements made between the two images and taken as one measure of performance [5].

Detailed recording of one UK expert's visual search behaviour over a number of MLO view screening cases has elucidated somewhat different search behaviour (e.g. Figure $4,5)$ to that as advocated [6] and also quantifiably different from the radiography students (e.g. compare expert and student eye gaze examples for images A and B in figures 2, 3, 4 and 5) .

Here it was found that this experienced radiologist examined each breast in detail before comparisons were made between the two breasts. Clearly then, differences in saccadic eye movement patterns can be found between experienced radiologists; however it would be expected that there is similarity in the actual image areas foveally examined which should represent (1) known general high probability areas for abnormality presence (e.g. just behind the nipple) and (2) areas which, per image, suggest potential abnormal appearance based wholly on the individual appearance of that image. This is supported by a recent study [7] of 15 experienced screeners examining 40 cases which demonstrated that whilst each individual exhibited different scanning patterns yet they examined broadly similar image areas in detail.

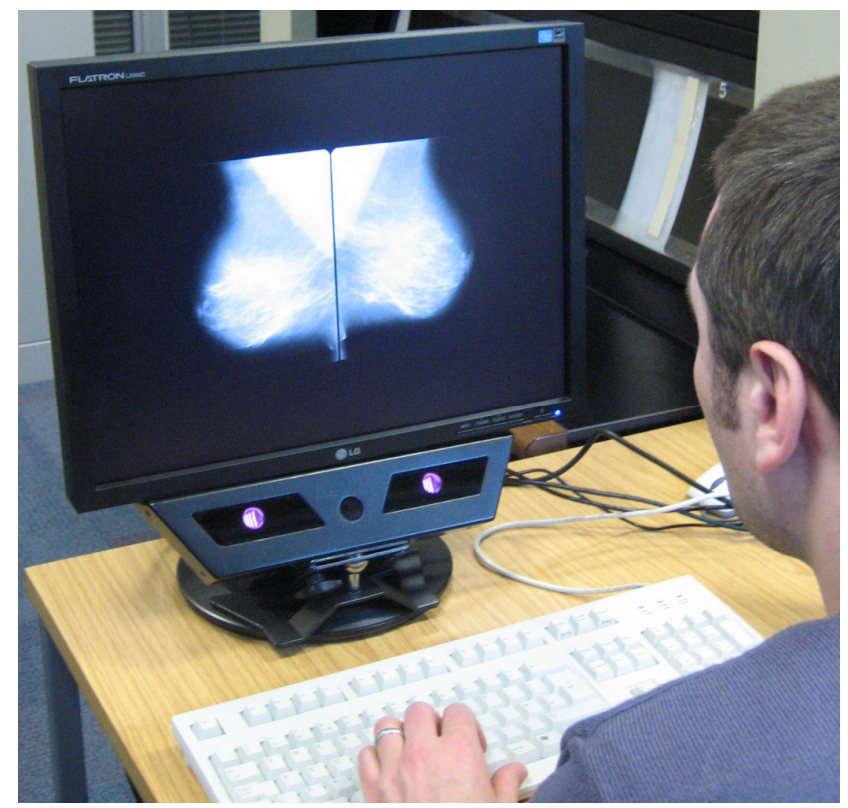

Figure 1. The experimental set up 

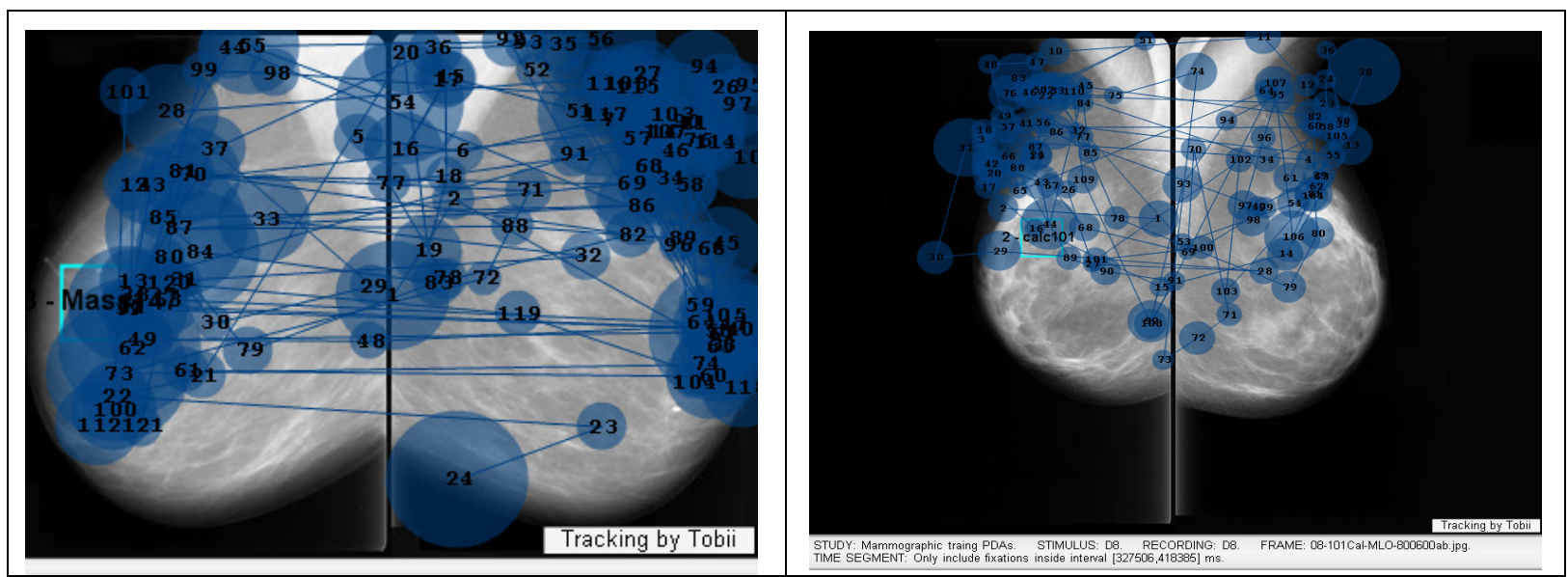

Figure 2. Student observer examining image A

Figure 3. Student observer examining image B
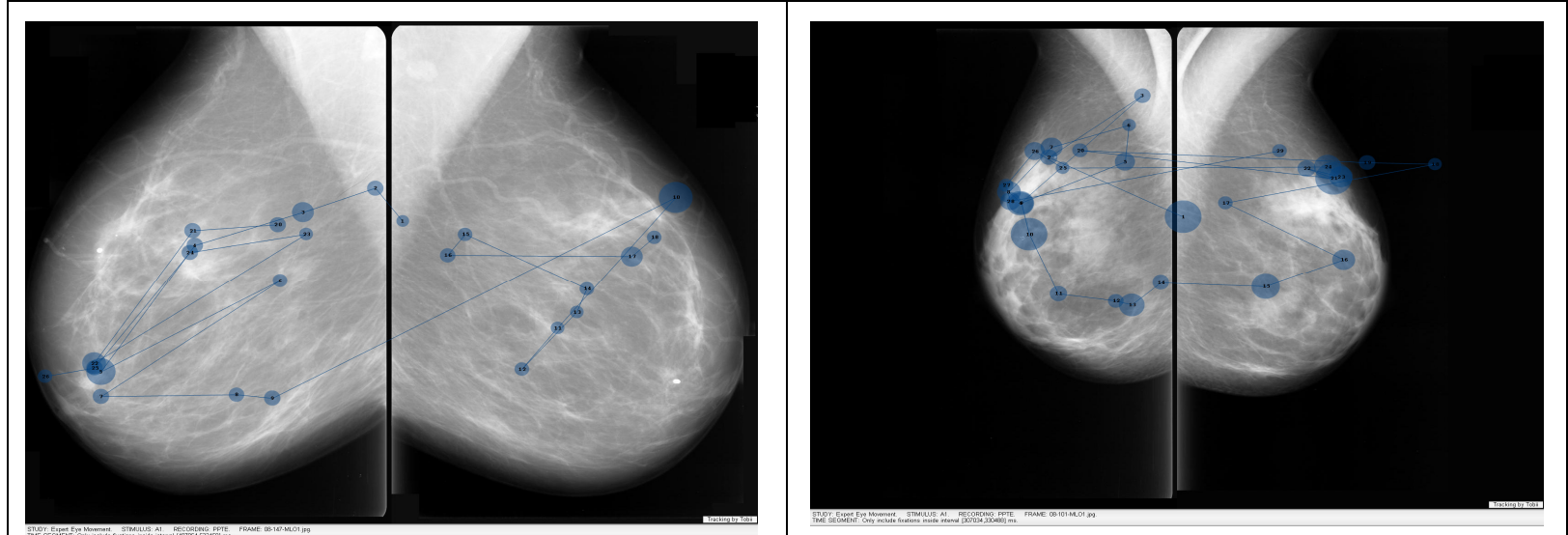

Figure 4. Expert radiologist examining image A

Figure 5. Expert radiologist examining image B
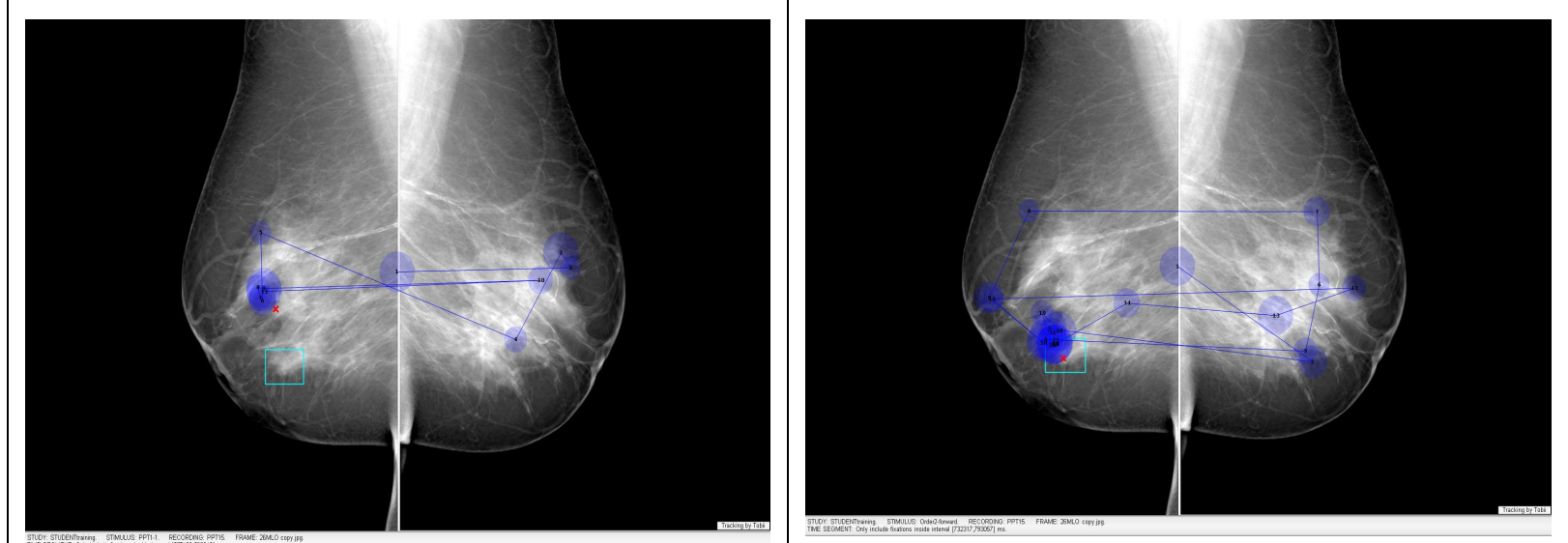

Figure 6. Naïve observer before training

Figure 7. Naïve observer after training 


\section{VISUAL GAZE AS A TRAINING TOOL}

An experimental investigation was carried out [6] to examine potentially how to utilise an expert's visual search behaviour as a possible tool for mammographic interpretation training. Analysis of an expert's visual search data over 21 cases allowed identification of regions of interest around particular cancers and also around other image areas which attracted visual attention but were not abnormal.

As part of a larger study [6], eight naive observers, with no knowledge of radiology, were first given a short standardised introductory computer-based presentation about breast cancer; mammograms, and the appearances of two key mammographic features - masses and calcifications. They were then split into a training and a control group. For both groups, each participant was tested twice, which involved identifying whether a series of breast screening cases demonstrated cancer. While they were examining the cases, participants' eye-movements were recorded. For the one hour break between the tests, the training group undertook the training package, which presented MLO mammograms incorporating a playback of the expert's visual search behaviour; and then regions of interest, as identified by the expert, were highlighted. For the control group no training was undertaken (see Figure 8).

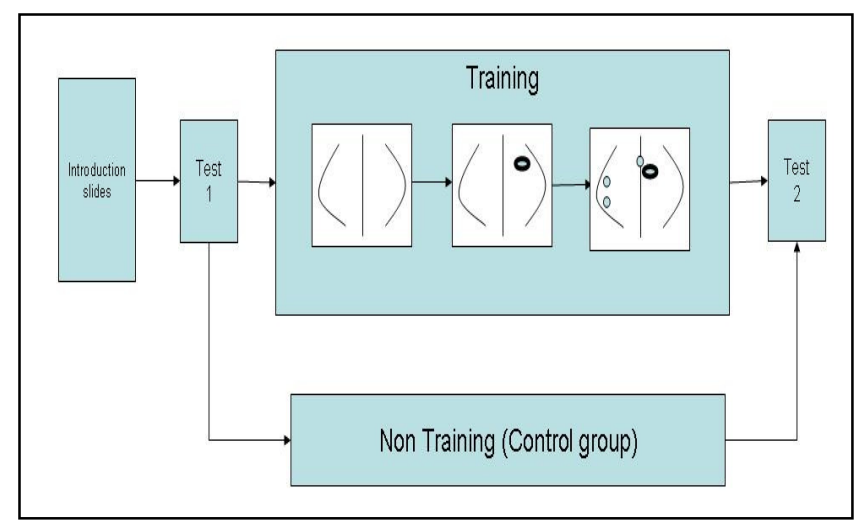

Figure 8. Schematic of the training

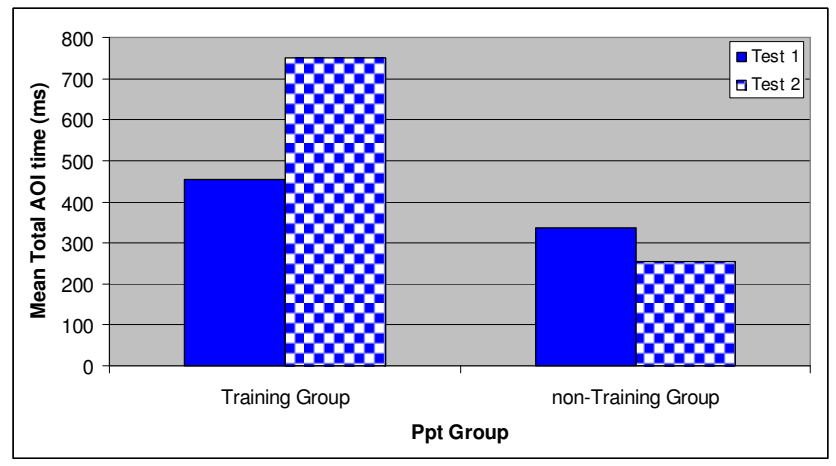

Figure 9. Mean times within AOIs for the abnormal images
From the eye movement records it was apparent that even after such a short training session the trained naïve observers were able to use an ordered search strategy which resulted in them fixating for longer time within an area of interest around the abnormality (Figure 9). As would be expected here, most errors were due to visual search and whilst errors decreased after training this was not significant $(\mathrm{p}>.05)$. Figures 6 and 7 shows a naïve observer examining the same image, before and after training where in the latter they identified the abnormality and spent time within the AOI ( as shown by the square in the images). Clearly simply following the expert's search behaviour is not, of itself, sufficient to improve performance quickly. However from such data it is possible to determine key areas which attract an expert's visual attention and coupling this with knowledge of abnormality appearance could offer additional training benefits. Such an approach could also be married to employing CAD in training.

\section{CONCLUSIONS}

Breast screening is a key imaging domain requiring highly trained observers. It is proposed here that such training could well utilise aspects of CAD where algorithms identify potential abnormal areas, coupled with also employing expert observers' eye gaze to further indicate to trainees those potential suspicious image areas which may be indicative of abnormality. Combining these two approaches would offer a new practical application incorporating eye gaze into intelligent machine interaction to produce enhanced training systems for breast cancer screening.

\section{REFERENCES}

1. Kundel H.L. Nodine C.F. Carmody D. Visual Scanning, Pattern Recognition and Decision-making in Pulmonary Nodule Detection. Investigative Radiology (1978), 13(3):175-181.

2. Gilbert F.J., Astley S.M., et al. Single Reading With Computer-Aided Detection For Screening Mammography. N Engl J Med (2008) 16:1675-84.

3. Chen Y., Gale A.G., Scott H. Mammographic interpretation training: how useful is handheld technology? In Proc. SPIE Medical Imaging 2008: SPIE Vol. 7263.

4. Tabar L., Dean P.B. Teaching Atlas of Mammography. (Ed 3). New York: Thieme, 2000.

5. Mugglestone M D, Gale A G, Wilson A R M Perceptual processes involved in mammographic film interpretation, In Proc. SPIE Medical Imaging 1997: SPIE Vol. 3036.

6. Chen Y., Gale A.G., Breast screening: a vision supported healthcare training approach. In Proc. IEA 2009

7. Chen Y., Gale A.G., et al. Breast Screening: visual search as an aid for digital mammographic interpretation training. In Proc. SPIE Medical Imaging (2010). In Press. 